\title{
Revealing Three-Dimensional Structure of an Individual Colloidal Crystal Grain by Coherent X-Ray Diffractive Imaging
}

\author{
A. G. Shabalin, ${ }^{1,2}$ J.-M. Meijer, ${ }^{3, \dagger}$ R. Dronyak, ${ }^{1}$ O. M. Yefanov, ${ }^{1,}$ A. Singer, ${ }^{1,}$ R. P. Kurta, ${ }^{1,}$ \\ U. Lorenz, ${ }^{1}$ O. Y. Gorobtsov, ${ }^{1,4}$ D. Dzhigaev, ${ }^{1,5}$ S. Kalbfleisch, ${ }^{6}$ J. Gulden, ${ }^{1}$ A. V. Zozulya, ${ }^{1}$ \\ M. Sprung, ${ }^{1}$ A. V. Petukhov, ${ }^{3,7}$ and I. A. Vartanyants ${ }^{1,5,}$ \\ ${ }^{1}$ Deutsches Elektronen-Synchrotron DESY, Notkestr. 85, D-22607 Hamburg, Germany \\ ${ }^{2}$ A.V. Shubnikov Institute of Crystallography RAS, Leninskii pr. 59, 119333 Moscow, Russia \\ ${ }^{3}$ Van 't Hoff laboratory for Physical and Colloid chemistry, Debye Institute for Nanomaterials Science, Utrecht University, \\ Padualaan 8, 3508 TB Utrecht, Netherlands \\ ${ }^{4}$ NRC Kurchatov Institute, pl. Akademika Kurchatova, Moscow 123182, Russia \\ ${ }^{5}$ National Research Nuclear University MEPhI (Moscow Engineering Physics Institute), \\ Kashirskoe shosse 31, 115409 Moscow, Russia \\ ${ }^{6}$ Institute for X-Ray Physics, Friedrich-Hund-Platz 1, 37077 Göttingen, Germany \\ ${ }^{7}$ Laboratory of Physical Chemistry, Department of Chemical Engineering and Chemistry, Eindhoven University of Technology, \\ P.O. Box 513, $5600 \mathrm{MB}$ Eindhoven, Netherlands
}

(Received 27 May 2016; published 22 September 2016)

\begin{abstract}
We present results of a coherent $\mathrm{x}$-ray diffractive imaging experiment performed on a single colloidal crystal grain. The full three-dimensional (3D) reciprocal space map measured by an azimuthal rotational scan contained several orders of Bragg reflections together with the coherent interference signal between them. Applying the iterative phase retrieval approach, the 3D structure of the crystal grain was reconstructed and positions of individual colloidal particles were resolved. As a result, an exact stacking sequence of hexagonal close-packed layers including planar and linear defects were identified.
\end{abstract}

DOI: 10.1103/PhysRevLett.117.138002

Colloidal crystals nowadays are actively exploited as an important model system to study nucleation phenomena in freezing, melting, and solid-solid phase transitions [1-5], jamming and glass formation [6,7]. In addition, colloidal crystals are attractive for multiple applications since they can be used as large-scale templates to fabricate novel materials with unique optical properties such as the full photonic band gap, "slow" photons and negative refraction, as well as materials for application in catalysis, biomaterials, and sensorics [8-11]. Colloidal crystals grown by self-organization provide a low cost large-scale alternative to lithographic techniques, which are very effective for producing high-quality materials with a desired structure, but are limited in building up a truly three-dimensional (3D) structure and bring high production costs [12]. Recently, significant progress has been achieved in engineering materials with tunable periodic structure on the mesoscale by functionalizing colloids with DNA [13], applying external fields [14,15] or varying the particle shape $[16,17]$.

Understanding the real structure of colloidal crystals and its disorder is an important aspect from both fundamental and practical points of view. Even at equilibrium, colloidal crystals can have a finite density of defects, which can be anomalously large for certain colloidal lattices [18]. The opposite can also happen as defects can play a decisive role in the choice of the crystal structure $[19,20]$. For applications such as photonic crystals most of growth-induced defects can deteriorate their optical properties. On the other hand, controlled incorporation of certain defects can be desirable to enhance the functionality such as creating wave guides [21,22], trapping photons [12,23], and developing optical chips [24]. In these studies, monitoring an internal 3D structure of colloidal crystals including defects in real time remains a challenge [25].

Among widely used techniques of the colloidal crystal structure investigation are optical microscopy [26,27] and confocal laser scanning microscopy [28]. However, the range of applications of these methods is strongly reduced by the limited resolution (at best about $250 \mathrm{~nm}$ ) and the need of a careful refractive index matching, which is not always possible. Furthermore, some of the materials are opaque for visible light which complicates imaging of their internal structure. Electron microscopy (EM) can provide high-quality images of the material surface with an exceptional resolution [29], but fails to probe the bulk, because of the short penetration depth of electrons. In addition, imaging in EM typically involves elaborate and destructive sample preparation [30], such as drying or coating. In this respect, high-resolution x-ray microscopy [31-34], smallangle $\mathrm{x}$-ray and neutron scattering $[35,36]$ represent complementary methods offering the advantage of high penetration depth for nondestructive studies of colloidal systems.

Here we present results of the coherent x-ray diffractive imaging (CXDI) approach [37,38] (see for review Ref. [39]), 
which allows us to visualize the internal 3D structure of an individual colloidal crystal grain with high resolution.

The original idea was proposed in a seminal work of Sayre [40], where he suggested to phase crystallographic data by measuring information between the Bragg peaks [41]. This concept applied for 3D imaging of the internal structure of crystalline samples can, in principle, provide information about the positions of individual scatterers in the crystalline structure [39]. We have demonstrated in simulations that such an approach may be applied to atomic resolution imaging of nanocrystals illuminated with high energy coherent $x$ rays by simultaneous mapping of several Bragg peaks [43] (similar to electron diffraction [44-46]). However, lack of coherent flux as well as low scattering efficiency at these energies are strong limiting factors to reach these goals presently.

Colloidal crystals with the unit cell on the order of a few hundred nanometers are ideal objects for developing these high resolution imaging methods. The first experimental demonstration of applying a two-dimensional (2D) CXDI method for visualization of a single stacking fault in a thin colloidal crystalline film was presented in Ref. [47]. Because of experimental challenges, attempts to generalize this approach to three dimensions were not successful so far [48]. Here we present the first successful realization of a detailed reconstruction, visualizing 3D positions of individual particles in a single colloidal crystal grain.

The experiment was performed at the Coherence Beam line P10 at PETRA III in Hamburg. A monochromatic coherent $\mathrm{x}$-ray beam of $8 \mathrm{keV}$ photon energy was focused at the sample at $87.7 \mathrm{~m}$ from the undulator source using the transfocator optics [49] based on compound refractive lenses (CRLs) positioned at $2.2 \mathrm{~m}$ distance upstream from the sample (see Fig. 1). A set of horizontal and vertical guard slits $75 \times 75 \mu \mathrm{m}^{2}$ in size, located in front of the CRLs, was used to select a coherent portion of the beam. The size of the focal spot at the sample position was $5.5 \times 3.2 \mu \mathrm{m}^{2}$ full width at half maximum (FWHM) in the horizontal and vertical directions, with the total intensity about $10^{11}$ photons per second [49]. The sample holder was mounted on a goniometer, which allows azimuthal rotation around the vertical axis. The diffraction data were recorded using a photon-counting pixel detector MAXIPIX positioned in transmission geometry at $5.1 \mathrm{~m}$ distance downstream from the sample. The total number of pixels of the detector was $516 \times 516$ and a pixel size was $55 \times 55 \mu \mathrm{m}^{2}$. At the selected photon energy and sample-to-detector distance resolution in reciprocal space was $0.437 \mu \mathrm{m}^{-1}$ per pixel and afforded 4 times sampling rate per speckle. The signal scattered up to an edge of the detector provided real space resolution of about $56 \mathrm{~nm}$.

Samples were prepared from dried sediments of colloidal crystals that showed characteristic optical Bragg reflections. Our specimen consisted of sterically stabilized silica spheres with a diameter of $230 \mathrm{~nm}$ dried from cyclohexane

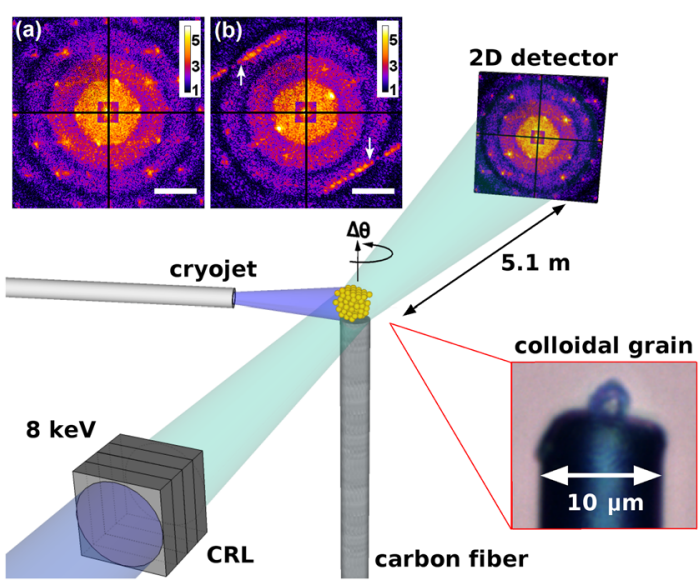

FIG. 1. Schematic layout of the experimental setup. Compound refractive lenses (CRL) focus the coherent $\mathrm{x}$-ray beam at the crystal grain mounted on a top of the carbon fiber (light microscope image is shown in lower right inset). The sample was constantly cooled by a flow of nitrogen using a cryojet. The diffraction data were recorded by a $2 \mathrm{D}$ detector positioned in the far field. (a),(b) Typical diffraction patterns in logarithmic scale measured at relative angular positions $\Delta \theta=61^{\circ}$ (a) and $\Delta \theta=$ $165^{\circ}$ (b). Two elongated rods visible in (b) (indicated by arrows) originate from the presence of a planar defect in the crystal grain. Scale bar in $(\mathrm{a}, \mathrm{b})$ is $50 \mu \mathrm{m}^{-1}$.

over several months. Small grains were obtained by mechanically crushing a piece of the ordered sediment. Individual grains were picked up using a micromanipulator and connected to the tip of a $10 \mu \mathrm{m}$ thick carbon fiber, which was glued to a glass holder. The colloidal crystal grain used in this study was imaged with a light microscope [50] (see inset in Fig. 1) and determined to have dimensions of about $2 \times 3 \times 4 \mu \mathrm{m}^{3}$.

The full data set consisted of a rotation series of 360 diffraction patterns with $0.5^{\circ}$ angular increment covering the entire reciprocal space. In Figs. 1(a), 1(b) two examples of measured diffraction patterns are shown. They contain several Bragg peaks surrounded by the interference speckles and diffuse scattering in between. The detector size allowed us to record reflections up to the fourth order. The visibility $V=\left(I_{\max }-I_{\min }\right) /\left(I_{\max }+I_{\min }\right)$, describing the contrast in the coherent interference pattern [55], was estimated to be from $75 \%$ to $80 \%$. It is worth mentioning that although the intensities between Bragg peaks are rather weak in comparison with the ones in the vicinity of Bragg peaks, this part of the recorded signal is highly important for the reconstruction. It encodes information about the relative phases of different reflections and allows us to resolve positions of individual scatterers in the unit cell. The concentric rings observed in diffraction patterns represent the form factor of a single colloidal sphere and the number of these rings gives an estimate of four pixels resolution per colloidal particle diameter in direct space.

The full 3D reciprocal space map, represented by volume rendering, is shown in Fig. 2(a). All 360 diffraction patterns 


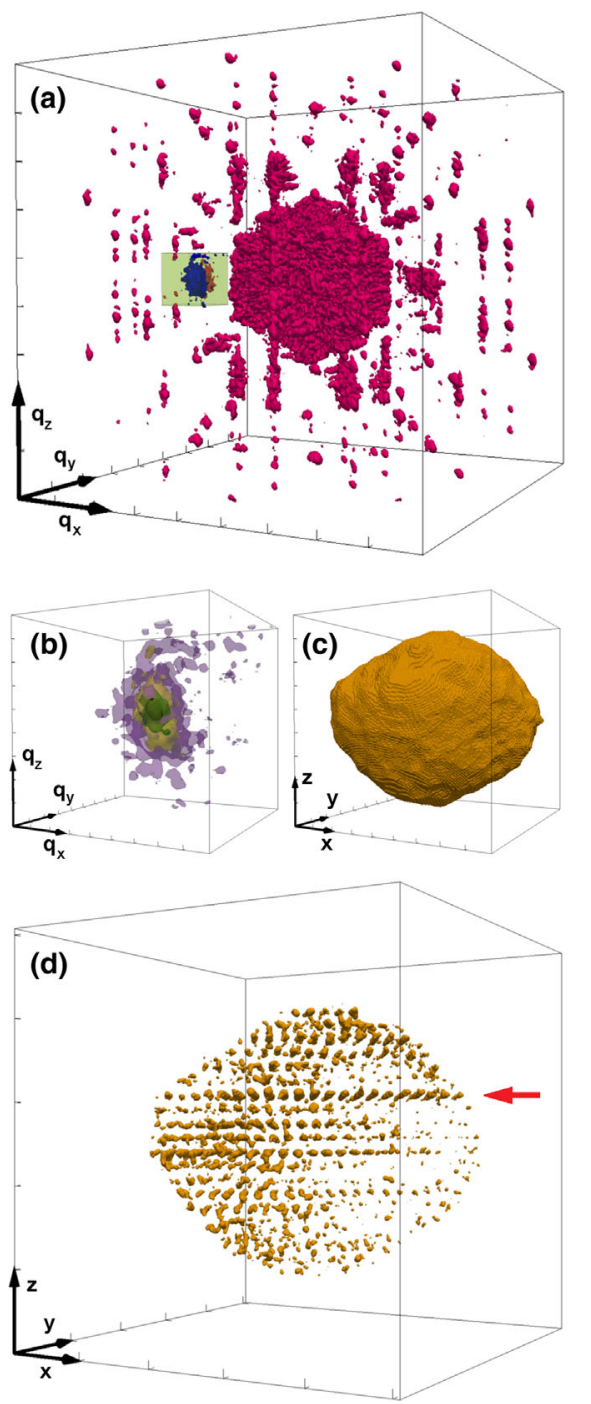

FIG. 2. (a) 3D scattered intensity in reciprocal space, represented by volume rendering. (b) Reciprocal space map in the vicinity of the Bragg peak shown by a green box in (a). (c) Averaged reconstruction of 3D shape of the grain in real space obtained from (b). (d) Reconstructed 3D electron density distribution in real space. The red arrow marks the crystalline plane shown as a 2D slice in Fig. 3(b). Length of the coordinate arrows correspond to $50 \mu \mathrm{m}^{-1}$ in (a), $10 \mu \mathrm{m}^{-1}$ in (b), and $1 \mu \mathrm{m}$ in (c),(d).

collected during the rotational scan were used in the merging procedure. A remarkable features observed in this map are a number of streaks which connect some of the Bragg peaks [see Fig. 2(a) and diffraction pattern in Fig. 1(b)]. These streaks (Bragg rods) indicate the presence of plane defects in the crystalline lattice and the intensity modulations along them are directly related to the exact stacking sequence [48,51]. In the full 3D reciprocal space data set [see Fig. 2(a)], that gives rise to well-pronounced Bragg rods which connect some of the reciprocal lattice nodes. Such rods are oriented perpendicular to the plane of the defect and therefore a specific sample orientation is required for the observation of the whole streak in a single diffraction pattern.

The 3D reciprocal space maps were inverted to real space images by using phase retrieval algorithms [56,57]. In order to obtain a good estimate for a tight support, which is crucial to enforce convergence of the reconstruction procedure, we analyzed the scattered intensity distribution in the vicinity of several Bragg reflections that were not affected by Bragg rods. From each of them we selected a cubic volume of $35 \times 35 \times 35 \mu \mathrm{m}^{-3}$ surrounding the Bragg peak [see Fig. 2(b)], and performed reconstruction for this cropped data set. The results were averaged over reconstructions of six different Bragg reflections, and the obtained shape function [see Fig. 2(c)] was used as a tight support in the reconstruction of the full 3D reciprocal space data set. That allowed us to avoid the appearance of twin images and substantially facilitated the reconstruction procedure. In the phase retrieval process, consisting of about 3000 iterations of Hybrid Input-Output (HIO) combined with Error Reduction (ER) algorithms [56], the support was several times updated by applying the Shrinkwrap method [58]. The missing regions in the diffraction data were allowed to freely evolve with an additional constraint of applying an upper boundary for amplitudes. This suppresses them down to values obtained from the Fourier transform of the support with normalization to the measured amplitudes.

Reconstructed 3D electron density after applying a high bandpass Gaussian filter is shown in Fig. 2(d). It reveals periodic behavior that corresponds to positions of colloidal particles in the crystal grain. In Fig. 3(b) a slice through one of the packing planes marked by a red arrow in Fig. 2(d) is presented. Remarkably, positions of individual colloidal spheres are clearly resolved and show perfect hexagonal symmetry. The distorted lattice and lower visibility near the crystal surface is not an artifact of reconstruction but is rather related to the real surface structure of the grain due to its preparation method [50].

We used the same slice to estimate an obtained resolution in real space for localization of individual particles in the crystal. An average density peak width in this image was estimated to be $70 \mathrm{~nm}$ (FWHM). Applying the Rayleigh criterion for the minimum separation between two distinguished Gaussian peaks we determined the resolution to be on the order of $80 \mathrm{~nm}$. At the same time, importantly, the position of each individual colloidal sphere in the lattice is determined with much higher accuracy of about $4 \%$ (or $9 \mathrm{~nm}$ ) due to the fact that several orders of Bragg peaks were measured simultaneously [50].

Furthermore, our results allow us to identify the stacking sequence of the colloidal crystal layers, which can be stacked in $A B C$ or $A B A$ manner as in face-centred cubic (fcc) or hexagonal close-packed (hcp) structures, respectively [see Fig. 3(a)]. Since for hard colloidal spheres the free-energy difference between fcc and hcp is rather small 
(a)
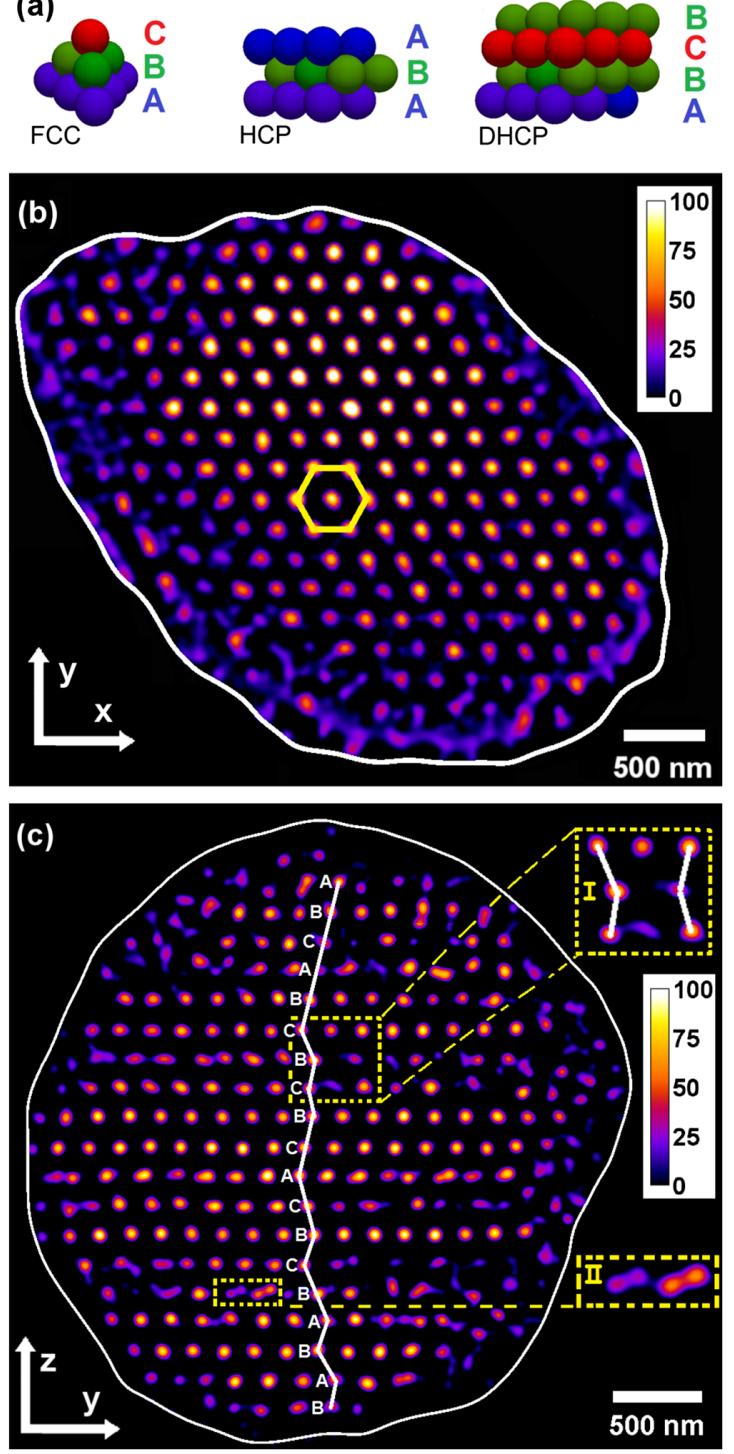

FIG. 3. (a) Examples of regular close-packed structures with the corresponding layer sequences: face centered cubic, hexagonal close packed, and double hexagonal close packed. (b) Slice through one of the packing planes. Well-pronounced hexagonal symmetry is outlined by a yellow hexagon. (c) Projection of the density map on the [100] crystallographic direction of the hexagonal lattice. Each layer is marked by the corresponding letter, the insets (I and II) show examples of in-plane defects.

[59], a random mixture of these two stacking types, the so-called random hexagonal close-packed (rhcp) structure, is often observed in colloidal crystals spontaneously self-assembled under gravity [60,61].

To determine the stacking sequence we analyzed the projection of the 3D density map along the [100] crystallographic direction of the hexagonal lattice defined in Ref. [51], i.e., along the $x$ axis in Fig. 2(d). To avoid error accumulation in the projection, each position of the intensity maxima in the 3D density profile was substituted by identical 3D Gaussian functions with the FWHM of
$70 \mathrm{~nm}$ and no density in between them. Next, for the sake of convenient visual representation, the resulting density map was divided by a 2D profile of the averaged intensity distribution, as a flat field correction to the image. In Fig. 3(c) each maximum represents a column of spherical particles in a crystalline arrangement. In the case of an ideal close-packed structure, projection of a column would correspond to projection of a single sphere. Nonuniform intensity distribution presented in Fig. 3(c) can be attributed to imperfections in positioning of individual particles (see Ref. [50]). Since the vector of lateral displacement belongs to the projection plane, the lateral position of each layer can be directly identified. The first well-pronounced layer at the top of the crystal was denoted as an $A$ layer and the next layer below as a $B$ layer. Then, according to its lateral shift, the third layer appears to be a $C$ layer. Following this procedure, 19 layers in the colloidal crystal were identified [see Fig. 3(c)]. One of the possible sequences of elementary stacking order may be $\underbrace{A B C} \underbrace{A B C B} \underbrace{C B C A} \underbrace{C B} \underbrace{C B A B} \underbrace{A B}$ (from top to bottom) that reveals three packing structures: fcc, hcp, and double hexagonal close-packed (dhcp) structure. The last one is usually described by $A B C B$ layer period [see Fig. 3(a)]. Here we proposed one of the possible sequences of the elementary stacking order; however, alternative decompositions are also possible. Still, all of them will contain fcc, hcp, and dhcp blocks in different combinations.

In addition to regular structures revealed by our analysis, we have observed a number of linear defects in the projection of 3D colloidal crystal grain [see insets in Fig. 3(c)]. In inset I it is clearly seen that at this specific location the left part of the crystal sequence can be clearly identified as $B$ position, while the right part is rather $A$ position [52]. In the inset II, a different type of defect is shown, when several double maxima appear in the projection. Our inspection showed that it can be attributed to the boundary between two perfect hexagonal regions, which are shifted by one-third of the period perpendicular to the projection direction. Thus, our reconstruction visualizes the exact stacking sequence of layers and in-plane defects in colloidal crystal grain. This is an important achievement which opens a way to further applications of the CXDI method for nondestructive characterization of photonic crystals.

In summary, the nondestructive CXDI experiment on a single colloidal crystal grain was performed. Full crystallographic data including several Bragg reflections, surrounding speckles, and intensities between them was measured by a rotation series of 2D far-field diffraction patterns. By applying the phase retrieval approach, the obtained 3D data set in reciprocal space was inverted into the electron density distribution in real space. Finally, the grain shape and positions of individual colloidal particles, resolved in three dimensions, were reconstructed. The crystalline structure of the sample was characterized in terms of close packing of hexagonal layers. The determined stacking sequence 
revealed 19 layers with fcc, hcp, and dhcp structure blocks. Our experiment allowed us to visualize with high resolution a number of in-plane defects [52] present in the colloidal crystal grain, including all the details up to its core. Development of the technique could provide, in the future, important statistical information about the strain deformation around a defect [62].

Our results open up a breakthrough in applications of coherent $\mathrm{x}$-ray diffraction for visualization of the inner three-dimensional structure of different mesoscopic materials, such as photonic crystals. The outcome of this work is of significant importance for further progress and developments of CXDI methods with an aim to resolve the 3D structure of colloidal crystals with smaller particle size [50] and nanocrystals with atomic resolution [43]. Our achievements pave the way to atomic resolution imaging of nanocrystals at the next generation diffraction limited synchrotron light sources [63], which are expected to provide 2 orders of magnitude higher brilliance and coherent flux.

This research was supported by BMBF Proposal 05K10CHG "Coherent Diffraction Imaging and Scattering of Ultrashort Coherent Pulses with Matter" in the framework of the German-Russian collaboration Development and Use of Accelerator-Based Photon Sources and the Virtual Institute VH-VI-403 of the Helmholtz Association. The use of the GINIX setup operated by the University of Göttingen is greatly acknowledged. We acknowledge fruitful discussions and support of the project by E. Weckert, important comments and suggestions on the final stages of the manuscript preparation by S. Lazarev, and careful reading of the manuscript by $\mathrm{D}$. Novikov.

*Corresponding author.

ivan.vartaniants@desy.de

${ }^{\dagger}$ Present address: Division of Physical Chemistry, Department of Chemistry, Lund University, 22100 Lund, Sweden.

*Present address: Center for Free-Electron Laser Science, DESY, Notkestr. 85, 22607 Hamburg, Germany.

${ }^{\S}$ Present address: University of California-San Diego, 92093 La Jolla, USA.

Tresent address: European XFEL GmbH, Holzkoppel 4, D-22869 Schenefeld, Germany.

[1] W. Qi, Y. Peng, Y. Han, R. K. Bowles, and M. Dijkstra, Phys. Rev. Lett. 115, 185701 (2015).

[2] Z. Wang, F. Wang, Y. Peng, and Y. Han, Nat. Commun. 6, 6942 (2015).

[3] A. Statt, P. Virnau, and K. Binder, Phys. Rev. Lett. 114, 026101 (2015).

[4] Y. Peng, F. Wang, Z. Wang, A. M. Alsayed, Z. Zhang, A. G. Yodh, and Y. Han, Nat. Mater. 14, 101 (2015).

[5] B. Cabane, J. Li, F. Artzner, R. Botet, C. Labbez, G. Bareigts, M. Sztucki, and L. Goehring, Phys. Rev. Lett. 116, 208001 (2016).

[6] E. DeGiuli, E. Lerner, C. Brito, and M. Wyart, Proc. Natl. Acad. Sci. U.S.A. 111, 17054 (2014).
[7] A. R. Jacob, A.S. Poulos, S. Kim, J. Vermant, and G. Petekidis, Phys. Rev. Lett. 115, 218301 (2015).

[8] S.-H. Kim, S. Y. Lee, S.-M. Yang, and G.-R. Yi, NPG Asia Mater. 3, 25 (2011).

[9] A. Kuzyk, R. Schreiber, Z. Fan, G. Pardatscher, E.-M. Roller, A. Högele, F. C. Simmel, A. O. Govorov, and T. Liedl, Nature (London) 483, 311 (2012).

[10] E. Henry, A. Dif, M. Schmutz, L. Legoff, F. Amblard, V. Marchi-Artzner, and F. Artzner, Nano Lett. 11, 5443 (2011).

[11] Y. Zhang, F. Lu, K. G. Yager, D. van der Lelie, and O. Gang, Nat. Nanotechnol. 8, 865 (2013).

[12] O. Painter, R. K. Lee, A. Scherer, A. Yariv, J. D. O'brien, P. D. Dapkus, and I. Kim, Science 284, 1819 (1999).

[13] C. Zhang, R. J. Macfarlane, K. L. Young, C. H. J. Choi, L. Hao, E. Auyeung, G. Liu, X. Zhou, and C. A. Mirkin, Nat. Mater. 12, 741 (2013).

[14] A. Yethiraj and A. van Blaaderen, Nature (London) 421, 513 (2003).

[15] A. Pal, V. Malik, L. He, B. H. Erné, Y. Yin, W. K. Kegel, and A. V. Petukhov, Agew. Chem., Int. Ed. 127, 1823 (2015).

[16] S. C. Glotzer and M. J. Solomon, Nat. Mater. 6, 557 (2007).

[17] A. V. Petukhov, J.-M. Meijer, and G. J. Vroege, Curr. Opin. Colloid Interface Sci. 20, 272 (2015).

[18] F. Smallenburg, L. Filion, M. Marechal, and M. Dijkstra, Proc. Natl. Acad. Sci. U.S.A. 109, 17886 (2012).

[19] N. A. Mahynski, A. Z. Panagiotopoulos, D. Meng, and S. K. Kumar, Nat. Commun. 5, 4472 (2014).

[20] J. Hilhorst, J. R. Wolters, and A. V. Petukhov, CrystEngComm 12, 3820 (2010).

[21] S. G. Johnson and J. D. Joannopoulos, Photonic Crystals: The Road from Theory to Practice (Springer, New York, 2002).

[22] J. Hilhorst, V. V. Abramova, A. Sinitskii, N. A. Sapoletova, K. S. Napolskii, A. A. Eliseev, D. V. Byelov, N. A. Grigoryeva, A. V. Vasilieva, W. G. Bouwman, K. Kvashnina, A. Snigirev, S. V. Grigoriev, and A. V. Petukhov, Langmuir 25, 10408 (2009).

[23] Y. A. Vlasov, X.-Z. Bo, J. C. Sturm, and D. J. Norris, Nature (London) 414, 289 (2001).

[24] M. Soljačić, C. Luo, J. D. Joannopoulos, and S. Fan, Opt. Lett. 28, 637 (2003).

[25] E. A. Sulyanova, A. Shabalin, A. V. Zozulya, J.-M. Meijer, D. Dzhigaev, O. Gorobtsov, R. P. Kurta, S. Lazarev, U. Lorenz, A. Singer et al., Langmuir 31, 5274 (2015).

[26] A. D. Dinsmore, E. R. Weeks, V. Prasad, A. C. Levitt, and D. A. Weitz, Appl. Opt. 40, 4152 (2001).

[27] P. Schall, I. Cohen, D. A. Weitz, and F. Spaepen, Science 305, 1944 (2004).

[28] P. Schall, Rep. Prog. Phys. 72, 076601 (2009).

[29] P. Jiang, J. F. Bertone, K. S. Hwang, and V. L. Colvin, Chem. Mater. 11, 2132 (1999).

[30] Y.-H. Ye, T. S. Mayer, I.-C. Khoo, I. B. Divliansky, N. Abrams, and T. E. Mallouk, J. Mater. Chem. 12, 3637 (2002).

[31] A. Bosak, I. Snigireva, K. S. Napolskii, and A. Snigirev, Adv. Mater. 22, 3256 (2010).

[32] J. Hilhorst, M. M. van Schooneveld, J. Wang, E. de Smit, T. Tyliszczak, J. Raabe, A. P. Hitchcock, M. Obst, F. M. F. de Groot, and A. V. Petukhov, Langmuir 28, 3614 (2012). 
[33] D. V. Byelov, J.-M. Meijer, I. Snigireva, A. Snigirev, L. Rossi, E. van den Pol, A. Kuijk, A. Philipse, A. Imhof, A. van Blaaderen, G. J. Vroege, and A. V. Petukhov, RSC Adv. 3, 15670 (2013).

[34] M. M. van Schooneveld, J. Hilhorst, A. V. Petukhov, T. Tyliszczak, J. Wang, B. M. Weckhuysen, F. M. F. de Groot, and E. de Smit, Small 7, 804 (2011).

[35] E. B. Sirota, H. D. Ou-Yang, S. K. Sinha, P. M. Chaikin, J. D. Axe, and Y. Fujii, Phys. Rev. Lett. 62, 1524 (1989).

[36] L. B. Chen, M. K. Chow, B. J. Ackerson, and C. F. Zukoski, Langmuir 10, 2817 (1994).

[37] J. Miao, P. Charalambous, J. Kirz, and D. Sayre, Nature (London) 400, 342 (1999).

[38] I. K. Robinson, I. A. Vartanyants, G. J. Williams, M. A. Pfeifer, and J. A. Pitney, Phys. Rev. Lett. 87, 195505 (2001).

[39] I. A. Vartanyants and O. M. Yefanov, X-ray Diffraction: Modern Experimental Techniques, edited by O. H. Seeck and B. Murphy (Pan Stanford Publishing, Singapore, 2015), Chap. 12, p. 341.

[40] D. Sayre, Acta Crystallogr. 5, 60 (1952).

[41] Demonstration of this approach to reveal the structure of a single protein was given in a recent work performed at freeelectron lasers [42].

[42] K. Ayyer, O. M. Yefanov, D. Oberthür, S. Roy-Chowdhury, L. Galli, V. Mariani, S. Basu, J. Coe, C. E. Conrad, R. Fromme et al., Nature (London) 530, 202 (2016).

[43] J. Gulden, O. M. Yefanov, E. Weckert, and I. A. Vartanyants, AIP Conf. Proc. 1365, 42 (2011).

[44] J. M. Zuo, I. A. Vartanyants, M. Gao, R. Zhang, and L. A. Nagahara, Science 300, 1419 (2003).

[45] R. Dronyak, K. S. Liang, J.-S. Tsai, Y. P. Stetsko, T.-K. Lee, and F.-R. Chen, Appl. Phys. Lett. 96, 221907 (2010).

[46] C.-C. Chen, C. Zhu, E. R. White, C.-Y. Chiu, M. C. Scott, B. C. Regan, L. D. Marks, Y. Huang, and J. Miao, Nature (London) 496, 74 (2013).

[47] J. Gulden, O. M. Yefanov, A. P. Mancuso, V. V. Abramova, J. Hilhorst, D. Byelov, I. Snigireva, A. Snigirev, A. V. Petukhov, and I. A. Vartanyants, Phys. Rev. B 81, 224105 (2010).
[48] J. Gulden, O. M. Yefanov, A. P. Mancuso, R. Dronyak, A. Singer, V. Bernátová, A. Burkhardt, O. Polozhentsev, A. Soldatov, M. Sprung, and I. A. Vartanyants, Opt. Express 20, 4039 (2012).

[49] A. V. Zozulya, S. Bondarenko, A. Schavkan, F. Westermeier, G. Grübel, and M. Sprung, Opt. Express 20, 18967 (2012).

[50] See Supplemental Material at http://link.aps.org/ supplemental/10.1103/PhysRevLett.117.138002 for the experimental details, stacking sequence determination, and road map for imaging colloidal crystals with smaller particles, which includes Refs. [51-54].

[51] J.-M. Meijer, A. Shabalin, R. Dronyak, O. M. Yefanov, A. Singer, R. P. Kurta, U. Lorenz, O. Gorobstov, D. Dzhigaev, J. Gulden, D. V. Byelov, A. V. Zozulya, M. Sprung, I. A. Vartanyants, and A. V. Petukhov, J. Appl. Crystallogr. 47, 1199 (2014).

[52] J.-M. Meijer, V. W. A. de Villeneuve, and A. V. Petukhov, Langmuir 23, 3554 (2007).

[53] P. Krishna and D. Pandey, Close-Packed Structures (IUCr University College Cardiff Press, Cardiff, 1981).

[54] N. Denkov, O. Velev, P. Kralchevski, I. Ivanov, H. Yoshimura, and K. Nagayama, Langmuir 8, 3183 (1992).

[55] J. W. Goodman, Introduction to Fourier Optics (Roberts and Co. Publishers, Greenwood Village, 2015).

[56] J. R. Fienup, Appl. Opt. 21, 2758 (1982).

[57] S. Marchesini, Rev. Sci. Instrum. 78, 011301 (2007).

[58] S. Marchesini, H. He, H. N. Chapman, S. P. Hau-Riege, A. Noy, M. R. Howells, U. Weierstall, and J. C. H. Spence, Phys. Rev. B 68, 140101 (2003).

[59] L. V. Woodcock, Nature (London) 385, 141 (1997).

[60] A. V. Petukhov, I. P. Dolbnya, D. G. A. L. Aarts, G. J. Vroege, and H. N. W. Lekkerkerker, Phys. Rev. Lett. 90, 028304 (2003).

[61] I. P. Dolbnya, A. V. Petukhov, D. G. A. L. Aarts, G. J. Vroege, and H. N. W. Lekkerkerker, Europhys. Lett. 72, 962 (2005).

[62] S. Ganguly, S. Sengupta, P. Sollich, and M. Rao, Phys. Rev. E 87, 042801 (2013).

[63] R. Hettel, J. Synchrotron Radiat. 21, 843 (2014). 